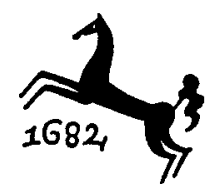

Germanistische ABHANDLUNGEN

Alfred Döblins historisches DenKen 


\title{
Alfred Döblins historisches Denken
}

\author{
Zur Poetik \\ des modernen Geschichtsromans
}

Mit einem Geleitwort von

Walter Müller-Seidel 
GERMANISTISCHE ABHANDLUNGEN 48

CIP-Kurztitelaufnahme der Deutschen Bibliothek

Wichert, Adalbert

Alfred Döblins historisches Denken: Zur Poetik d.

modernen Geschichtsromans. - 1. Aufl. - Stuttgart:

Metzler, 1978

(Germanistische Abhandlungen; 48)

ISBN 978-3-476-00377-5

ISBN 978-3-476-00377-5

ISBN 978-3-476-03087-0 (eBook)

DOI 10.1007/978-3-476-03087-0

(C) 1978 Springer-Verlag GmbH Deutschland

Ursprünglich erschienen bei J.B. Metzlersche Verlagsbuchhandlung und Carl Ernst Poeschel Verlag GmbH in Stuttgart 1978 


\section{GELEITWORT}

Den »Klassikern der Moderne «, wie man Hofmannsthal, Thomas Mann, Musil und andere Schriftsteller gelegentlich nennt, ist Alfred Döblin, der nunmehr »Hundertjährige «, offensichtlich nicht zuzurechnen. Ein Überblick über sein Werk wird dadurch erschwert, daß es eine auch nur annähernd vollständige Ausgabe nicht gibt. Nicht wenige seiner theoretischen Schriften sind längst vergriffen, und eine so gewichtige Erzählung aus der Frühzeit wie Der schwarze Vorhang, Roman von den Worten und Zufällen, hat vollends Seltenheitswert erhalten, im Buchhandel wie in unseren Bibliotheken. Dem gebildeten Leser ist das Erzählwerk Thomas Manns, Musils oder Kafkas geläufig und vertraut. Von Döblin kennt derselbe Leser allenfalls den Roman Berlin Alexanderplatz. Döblins chinesischer Roman (Die drei Sprünge des Wang-lun), der Wallenstein, die November-Trilogie, die Südamerikaromane oder die Erzählung mit dem aufregenden Titel Die Ermordung einer Butterblume sind ihm weithin unbekannte Texte. Dieser "Tatbestand « nimmt sich um so merkwürdiger aus, als es am geistigen und literarischen Rang dieses Autors keine Zweifel geben kann. Alfred Döblin ist ein Repräsentant der literarischen Moderne kat' exochen. Er ist es nicht zum geringsten aufgrund seiner vielseitigen Erfahrungen und Interessen. Als Naturforscher, Arzt und gesellschaftskundiger Denker kennt er sich in beiden Kulturen - der naturwissenschaftlichen wie der literarischen - aus eigener Anschauung aus. Das gibt auch seinem Geschichtsdenken wie seinen Geschichtserzählungen ihr besonderes Gewicht, und vorzüglich mit diesem Teilbereich des Gesamtwerks hat es die vorliegende Studie Adalbert Wicherts zu tun, der das Verdienst gebührt, einige neue Akzente gesetzt zu haben.

Dem Verfasser geht es vor anderem um den Nachweis, daß die bei Döblin hervortretende Geschichtskritik nicht als ein Symptom ahistorischen Denkens aufzufassen ist sondern ein anderes, von der Tradition des Historismus wegführendes Verständnis der geschichtlichen Welt bezeugt. Die Interpretation dieser kritischen Denkweise Döblins schließt Kritik des Verfassers an den Traditionen deutschen Geschichtsdenkens ein, wobei zu fragen bliebe, ob diese nicht ihrererseits auf historisches Verstehen Anspruch haben. In diesem Punkt kommt der Untersuchung partiell auch eine wissenschaftsgeschichtliche Bedeutung zu. Das wird eindringlich in dem Kapitel aufgezeigt, das von der "Entmythologisierung « der großen Männer handelt, die nach einem bekannten Spruch Geschichte machen. Hier vor allem hat das Buch einen seiner Schwerpunkte erhalten: im Aufweis einer gleichsam erzählten Sozialgeschichte, in der Handeln und Leiden ineinander übergehen, wie es zumal am Wang-lun sichtbar wird. Geschichtliches Handeln wird als soziales Handeln begriffen, aber zugleich werden Begriffe des frühen Expressionismus wie "aktiv" oder "Aktion " auf bezeichnende Weise umfunktioniert. Sie werden aufgrund der durchschauten Triebmechanismen als Formen des leidenden Menschen aufge- 
faßt. Es ist ein Vorzug dieser Arbeit, daß sie das veränderte Geschichtsdenken bis in seine sprachlichen Ausdrucksformen hinein verfolgt. Parataxe, Semantik des deformierten Menschen und anderes mehr erweisen sich als Äußerungen eines neuen Geschichtsdenkens, für das Schopenhauer als einer seiner Ahnherren wiederholt namhaft gemacht wird. In diesem Zusammenhang werden auch die weniger bekannten Romanwerke der späteren Zeit neu erschlossen und aufgewertet. Es ist zu hoffen, daß diese Studie der Forschung und daß sie dem Verständnis Döblins zugute kommt. Im Jahre seines 100. Geburtstages, in dem dieses Buch erscheint, wollen wir wünschen, daß sich möglichst viele diesen außerordentlichen und oft unbequemen Schriftsteller auf ihre Weise aneignen. 


\section{VORWORT}

Die vorliegende Studie wurde im Wintersemester 1976/77 vom Fachbereich Sprachund Literaturwissenschaft II der Universität München als Dissertation angenommen.

Mein Dank gilt allen Lehrern und Freunden, die die Arbeit begleitet haben. Den Mut zu beginnen hat mir Dr. Günter Hess gegeben. Entscheidende Hilfe war die engagierte Förderung durch Professor Dr. Walter Müller-Seidel, der mir bis zuletzt mit großem Interesse und mit seinem Rat beistand und mir dabei stets die Freiheit ließ, eigene Wege zu gehen.

Die Studienstiftung des deutschen Volkes hat die materielle Voraussetzung für die Entstehung dieses Buches geschaffen, für dessen sorgfältige und rasche Herstellung die J. B. Metzlersche Verlagsbuchhandlung und Dr. Uwe Schweikert gesorgt haben.

Der Dank an meine Eltern Kurt und Ursula Wichert läßt sich im Rahmen eines Vorworts ebenso wenig formulieren wie der Dank an Annemarie. Sie hat die Lasten der Entstehung gemeinsam mit mir getragen. Ihr sei dieses Buch gewidmet.

Augsburg, im November 1977

ADALBERT WICHERT 


\section{INHALTSVERZEICHNIS}

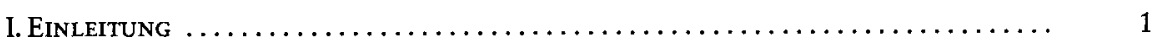

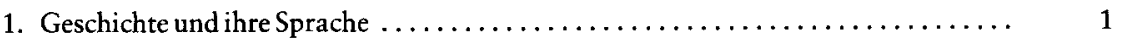

2. Döblins Interesse an der Geschichte und die Döblin-Forschung $\ldots \ldots \ldots \ldots \ldots \ldots \quad 4$

3. Jeder gute Roman ist ein historischer Roman $. \ldots \ldots \ldots \ldots \ldots \ldots \ldots \ldots . .6$

II. Der Angriff auf die tradittonelle Geschichtsvorstellung Und die ihr ENTSPRECHENDE TRADITIONELLE ERZÄHLWEISE $\ldots \ldots \ldots \ldots \ldots \ldots \ldots \ldots \ldots \ldots, 13$

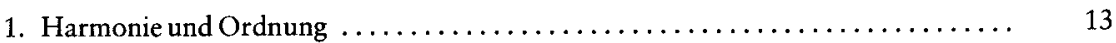

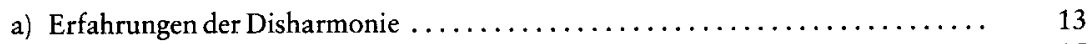

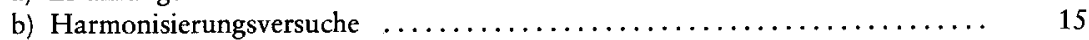

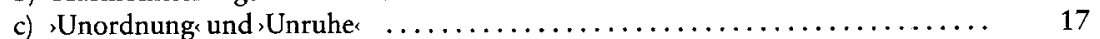

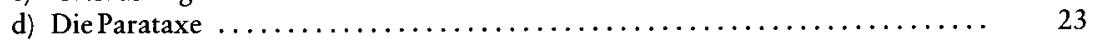

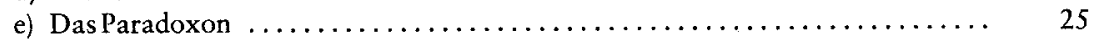

2. Kontinuität, Entwicklung und Fortschritt ..................... 27

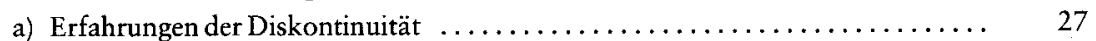

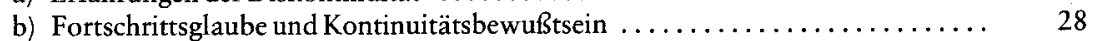

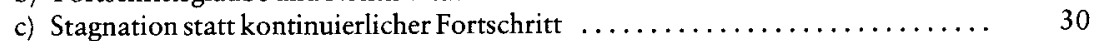

d) $H$ Hin und Her als Gegenmodell geschichtlichen Verlaufs . .............. 34

3. Erstarrung und Belebung in Geschichte und Erzählung . . . . . . . . . . . . 37

a) Die Verfestigung menschlicher Verhältnisse $\ldots \ldots \ldots \ldots \ldots \ldots \ldots \ldots \ldots . \ldots \ldots \ldots \ldots \ldots \ldots$

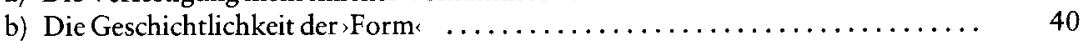

c) Die Befreiung des Epischen aus seiner erstarrten Form $\ldots \ldots \ldots \ldots \ldots \ldots .44$

d) Offene Geschichten $\ldots \ldots \ldots \ldots \ldots \ldots \ldots \ldots \ldots \ldots \ldots \ldots \ldots \ldots \ldots \ldots \ldots \ldots \ldots, \quad 51$

4. Parteilichkeit und Opposition als Formen antihistoristischen geschichtlichen Denkens .......................................... 54

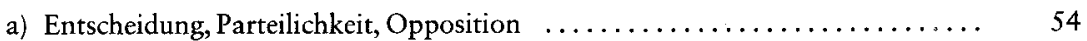

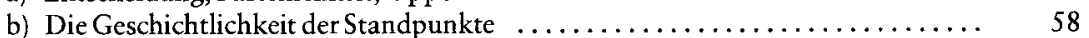

c) Die Revision des Wallensteinbildes .......................... 60

d) Oppositionelle Geschichtsschreibung $\ldots \ldots \ldots \ldots \ldots \ldots \ldots \ldots \ldots \ldots \ldots, \quad 70$

III. Die Konfrontation Der idealistischen Geschichtsauffassung mit deM

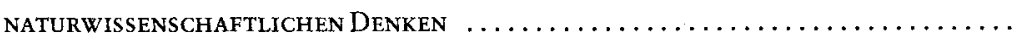

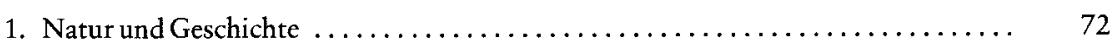

a) Die historische Bestimmung von Döblins Naturalismus ............. 72

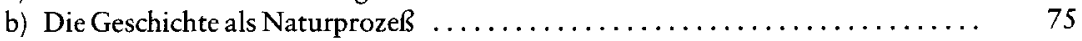

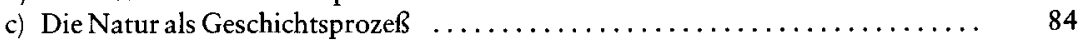

2. Bewußtsein und geschichtliche Erinnerung $\ldots \ldots \ldots \ldots \ldots \ldots \ldots \ldots \ldots \ldots \ldots, 87$

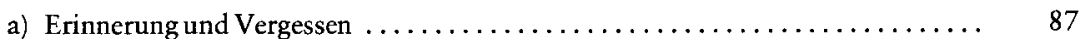

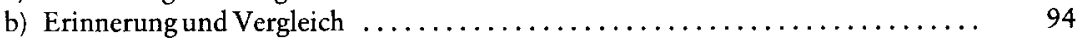




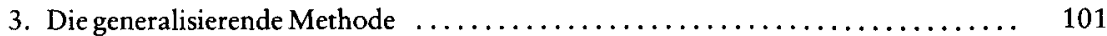

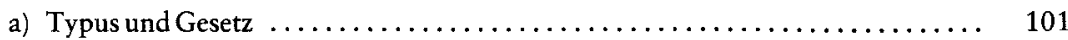

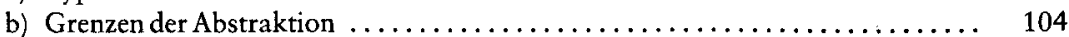

c) Die geschichtlichen Gesetze der Macht und der Freiheit ............. 109

d) Das Typische und die historische Situation $\ldots \ldots \ldots \ldots \ldots \ldots \ldots \ldots \ldots, 113$

IV. SozialgESCHICHTEN STATT HELDENGESCHICHTEN $\ldots \ldots \ldots \ldots \ldots \ldots \ldots \ldots \ldots \ldots \ldots$

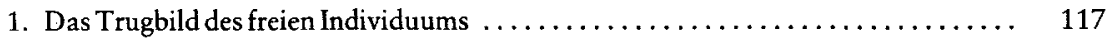

a) Der Kult der Vorbilder .................................. 117

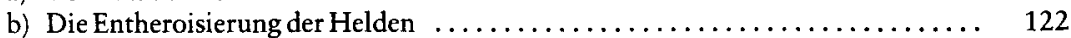

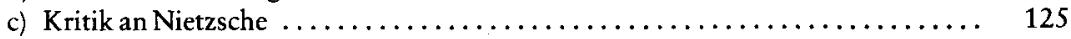

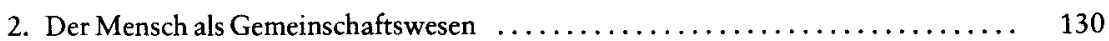

a) Die gesellschaftliche Natur des Menschen $\ldots \ldots \ldots \ldots \ldots \ldots \ldots \ldots \ldots .130$

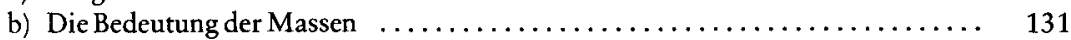

c) Herrschaft und Staat als Probleme der Gesellschaftsgeschichte ........... 145

d) Revolution und Religion $\ldots \ldots \ldots \ldots \ldots \ldots \ldots \ldots \ldots \ldots \ldots \ldots \ldots \ldots \ldots \ldots \ldots, 154$

V. Die Neubestimmung der geschichtlichen Handlung als SOziale Handlung .. 161

1. Die Ablösung des alten Tatbegriffs durch die Wechselwirkung $\ldots \ldots \ldots \ldots .161$

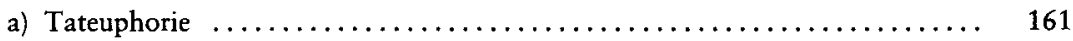

b) Die Widerlegung der individuellen Tatkraft durch Darstellung individueller Ohnmacht ....................................... 162

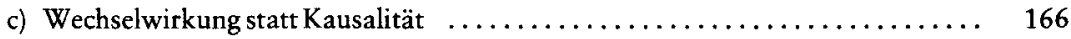

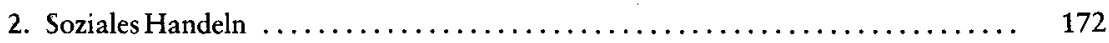

a) Das Schicksal als Summe von Bedingungen $\ldots \ldots \ldots \ldots \ldots \ldots \ldots \ldots \ldots . \ldots \ldots \ldots$

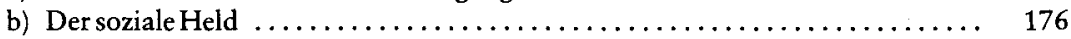

c) Leiden und Anpassung als Extremform situationsbedingten Handelns ....... 179

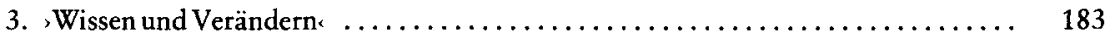

a) Das Erkennen der Bedingungen und Zusammenhänge als Form des Handelns .. 183

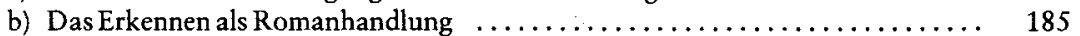

c) Geschichte als Universalgeschichte ......................... 190

d) Warten, Bewahren, Vorbereiten als Tätigkeit des Geistes $\ldots \ldots \ldots \ldots \ldots \ldots \quad 196$

e) Weitererzählen als Handeln $\ldots \ldots \ldots \ldots \ldots \ldots \ldots \ldots \ldots \ldots \ldots \ldots, 200$

VI. Das Erzählen von Geschichte als Wechselwirkung ZWischen Vergangenem

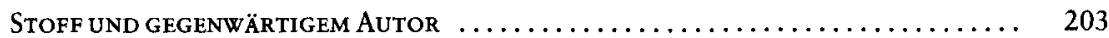

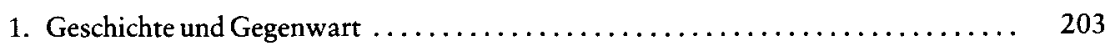

a) Kritik des Objektivitätsideals $\ldots \ldots \ldots \ldots \ldots \ldots \ldots \ldots \ldots \ldots \ldots \ldots \ldots, 203$

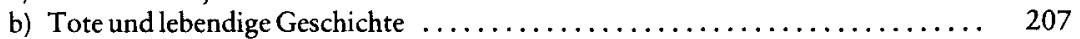

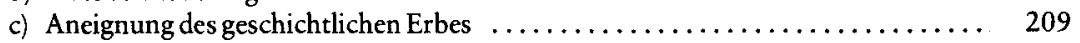

2. Der Mythos als Modell moderner Geschichten ..................... 214

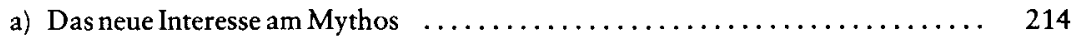

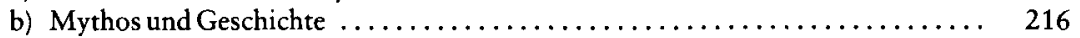

c) Der Erzähler und sein Stoff-Der Mythos als Erzählmodell $\ldots \ldots \ldots \ldots \ldots \ldots 220$

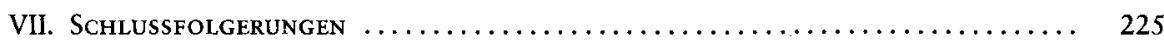

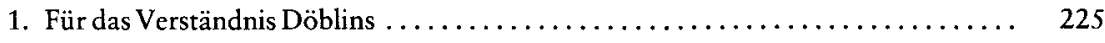

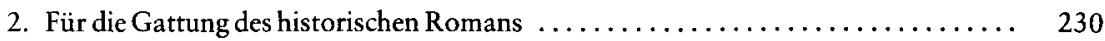


ANMERKUNGEN

LITERATURVERZEICHNIS 
"Wer diese Schulbücher passiert hat, weiß und hat und ist etwas vom gestrigen Deutschland, vom Kaiserreich, Mittelalter und Arnim. Die junge Republik, die Demokratie, die Arbeiterschaft, die neue Welt wird er nicht kennen, sondern ablehnen. [...] Die Frage der Zukunft wird sein: Wem gehört die Vergangenheit? «

Alfred Döblin (1936)

„Es kann - so meine ich - nicht um die Frage gehen: Tradition ja oder nein? Die Alternative besteht vielmehr darin, an welche Traditionen angeknüpft werden soll und in welchem Sinne wir eines historischen Vorganges gedenken. [...] Es ist Zeit, daß ein freiheitlich-demokratisches Deutschland unsere $\mathrm{Ge}$ schichte bis in die Schulbücher hinein anders schreibt. «

Gustav Heinemann (1970) 